\title{
In-situ measurements of temperature distribution during transmission laser welding of poly(aryletherketone)
}

\author{
M.Villar ${ }^{1}$, C.Garnier ${ }^{2}$, F.Chabert ${ }^{1, a)}$, V.Nassiet ${ }^{1}$, J.C.Diez ${ }^{3}$, A.Sotelo ${ }^{3}$, M.A.Madre ${ }^{3}$ \\ ${ }^{1}$ Interfaces and Functional Materials, LGP-ENIT-INPT, University of Toulouse, Tarbes, France \\ ${ }^{2}$ Mechanics of Materials, Structures and Processing, LGP-ENIT-INPT, University of Toulouse, Tarbes, France \\ ${ }^{3}$ Institute of materials science of Aragon, CSIC-University of Zaragoza, Spain

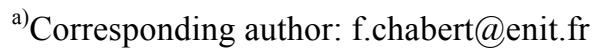

\begin{abstract}
In this work, the temperature distribution inside the specimens has been measured during transmission laser welding. The material studied is a high performance thermoplastic of the polyaryletherketone (PAEK) family. The assembly consists of a quasi-amorphous sample as the upper part and a semi-crystalline sample as the lower part. The temperature fields were measured by infrared thermography with the camera sensor perpendicular to the welded interface. With an energy beam of $28 \mathrm{~J} . \mathrm{mm}^{-2}$ and irradiation time of $15 \mathrm{~s}$, we have noticed that the maximum temperature inside the sample is kept far from the PAEK degradation one. Moreover, the temperature at the interface reaches the melting temperature thus assuring enough mobility for polymeric chains to get adhesion at the interface. Finally, the location and size of the heat-affected zone (HAZ) has been determined.
\end{abstract}

\section{INTRODUCTION}

Laser welding is an environmentally friendly and versatile process for assembling polymeric parts. Welding by transmission requires the upper element to be transparent to the laser wavelength whereas the inferior part is absorbent to the same wavelength. Thus, the energy of the beam is concentrated at the interface, allowing both polymeric parts to be melted. Upon temperature, the interdiffusion of polymeric chains takes place at the interface, creating bridges between both parts, resulting in a strong assembly. Laser welding is in principle applicable to any thermoplastic, provided that it is transparent to the laser wavelength. This process is already applied in the industry with common polymers such as polycarbonate and PMMA [1].

In some industrial fields such as aerospace and power electronics, only high performance polymers can be used. In some cases, these kinds of polymers gradually replace metallic alloys with the advantages to be lighter and corrosion resistant. Among them, the polyaryletherketone (PAEK) family has demonstrated to be the most resistant to thermo-oxidative degradation [2]. More knowledge and know-how about forming processes of PAEK would seemingly lead to widespread industrial uses.

Welding amorphous polymers is easier since the laser beam goes through the material without inducing major changes in the chemical structure and morphology of the material. From a theoretical point of view, the study of interdiffusion at the interface has been developed by the theory of reptation by De Gennes [3] and has been applied to the welding process in order to forecast the processing conditions [4], mainly temperature and time. Welding a semi-crystalline polymer is a challenge, since the upper part has to be transparent to the laser beam. As soon as the crystalline rate increases, any semi-crystalline polymer turns transparent to semi-transparent to opaque for the highest cristallinity grades.

Measuring the temperatures involved during laser welding is of great importance for many reasons: (a) checking that the temperature inside the polymeric part stays low enough to avoid both cold-crystallization and degradation of the material, (b) controlling the temperature to reach the melting temperature at the interface thus assuring enough mobility for polymeric chains to get adhesion and (c) knowing the location of the heat-affected zone (HAZ), the 
latter has possibly undergone morphological changes and thus, modification of properties and dimensions. In-situ temperature measurements would allow getting insight the laser welding process to make it more reliable.

Measuring the temperature at the interface is not easy because both polymeric parts are in contact all along the process. So, contact-free temperature measurement techniques such as infrared thermography have been implemented to the laser welding process in some studies $[5,6,7,8]$. In parallel to the experimental work, these authors have developed a numerical simulation to predict the temperature at the interface. To validate their model, the materials chosen are either PMMA as semi-transparent element and ABS/PC as absorbent part or, in another study, polycarbonate based FRP (fiber reinforced polymer). Both are amorphous polymers, with well-known transmission, reflection and absorption factors at near- and medium-IR spectrum. Although IR thermography seems to be suited to the transmission laser welding process, no study uses it as a technique for controlling temperatures distribution within the sample. In the existing studies, measurements were performed with the camera sensor parallel to the welded interface. With such an experimental setup, the temperature is measured at the upper or lower surface of the polymeric parts. These calculated/experimental results were implemented to numerical simulation, using a hybrid code that combines Mie theory [9,10] and the Monte Carlo method [11] to quantify the scattering phenomena. The experimental and numerical results match for the temperatures measured at the upper and lower surfaces, nevertheless, the temperature at the interface is forecast from these measurements but not really measured.

In this work, the feasibility of determining the temperature distribution at the interface within the sample during the welding process is shown. The temperature distribution is recorded by infrared thermography with the camera sensor perpendicular to the welded interface. Controlling the temperature distribution will allow to qualitatively predict the gradient of crystallization changes and so, changes in mechanical properties.

\section{MATERIALS AND METHODS}

A high performance semi-crystalline polyaryletherketone was chosen for this study. Two kinds of specimens were used for the welding test, an assembly made of a quasi-amorphous sample as the upper part and semicrystalline sample as the lower part. Their transmittance has been measured by spectrophotometer at the laser wavelength $(808 \mathrm{~nm})$ : It is $60 \%$ for the quasi-amorphous sample, whereas the transmittance of the semi-crystalline PAEK is less than 3\%. Based on these results, the name "semi-transparent" referred to the quasi-amorphous sample, and the name "opaque" referred to the semi-crystalline material in the following.

Both specimens were processed by compression molding from dried pellets. An $800 \mathrm{kN}$ Pinette Emidecau LAB $800 \mathrm{P}$ hot plates press was used; with controlled heating and cooling temperatures from $2^{\circ} \mathrm{C} / \mathrm{min}$ to $10^{\circ} \mathrm{C} / \mathrm{min}$. For controlling the crystalline rate and dimensions, samples were made in two steps. In the first step, the pellets were melted at $340^{\circ} \mathrm{C}$, slightly compressed and cooled down to room temperature. The cooling rate was $2^{\circ} \mathrm{C} \cdot \mathrm{min}^{-1}$ for the crystallized sample; and liquid nitrogen was used for a fast cooling rate to get the quasi-amorphous sample. In the second step, the sample were flattened up to $2 \mathrm{~mm}$ thick while heating at $2^{\circ} \mathrm{C} \cdot \mathrm{min}^{-1}$ until glass transition in order to remove any residual stress. The compression molded specimens were dried during $24 \mathrm{~h}$ at $140^{\circ} \mathrm{C}$ to remove water before testing.

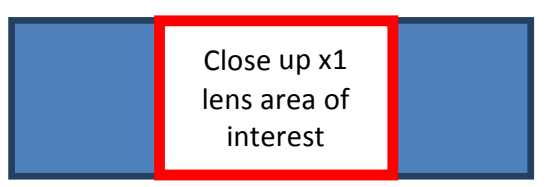

FIGURE 1: Infrared area of interest of the samples

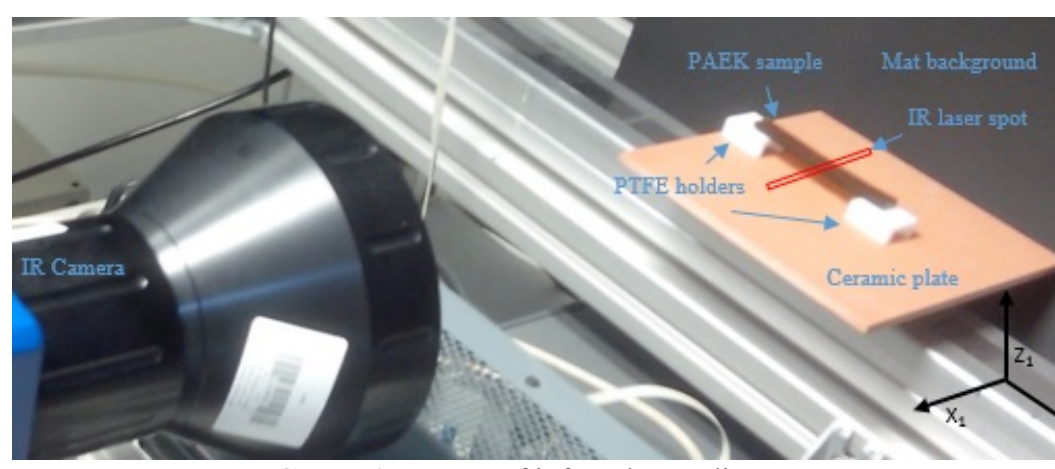

FIGURE 2: Layout of infrared recording scene 
DSC scan was performed with a DSC Q200 from TA Instruments, starting at room temperature up to $400^{\circ} \mathrm{C}$ with a ramp of $5^{\circ} \mathrm{C} / \mathrm{min}$. Only the quasi-amorphous compressed sample was studied. Glass transition temperature (Tg), cold crystallization temperature (Tcc) and melting temperature (Tm) were obtained.

Experimental near infrared laser device performs a continuous $808 \mathrm{~nm}$ beam, projecting a single $100 \times 1 \mathrm{~mm}^{2}$ spot. Laser source is fed by 90 diodes branched on 6 stacks of 15 diodes. Laser power is set by diode feeding intensity, starting power emission at $13 \mathrm{~A}$ and reaching $230 \mathrm{~W}$ for $40 \mathrm{~A}$. Nonetheless, power is spread out all along the laser spot, so only actual irradiated zone must be considered in interaction for energy calculations. Even though laser welding is normally performed in dynamic setup, static tests were carried out for a more accurate interfacial measurement, and to suppress parasite accumulative thermal effects appearing for dynamic laser welding.

Infrared temperature fields measurements were performed with an IR camera CEDIP ${ }^{\mathcal{O}}$ Jade III MWIR retrofitted FLIR $^{\circ}$ titanium SC 7200 with a thermal resolution of $20 \mathrm{mK}$ at $30^{\circ} \mathrm{C}$. The used detector is a mid-wave Indium Antimonide ( $\mathrm{InSb}$ ) one and the sensor is a close-up x1 covering a spectral range from 3.70 to $5.15 \mu \mathrm{m}$ with a focal plane array (FPA) windowing of $7.6 \times 9.9 \mathrm{~mm}^{2}$. In this range, the transmission factor has been measured by spectrophotometry: it is lower than $5 \%$ for both samples. Based on literature, we assume their reflectivity is much less than 1 [7] and so, the reflection factor is assumed to be less than $5 \%$. The detector resolution is 320x 256 pixels and the pitch of the sensor is $30 \mu \mathrm{m}$. So on, the pixel size on both directions is $0.03 \mathrm{~mm}$. Calibration and data recording were managed by Altair software.

Specimens were laid out $300 \mathrm{~mm}$ far from the close up X1 sensor to have the best sharpness. In order to measure interfacial temperature and the distribution of thermal field along both directions (thickness and length of the sample), side-by recordings were performed scoping the region of interest (Fig. 1). The whole experiments were done in a $25^{\circ} \mathrm{C}$ thermoregulated dark room to reduce the effect of environment on IR measurement. With regard to measure the rapid temperature rise during laser irradiation, integration time was set up at $16 \mu \mathrm{s}$, obtaining a temperature range from $140^{\circ} \mathrm{C}$ to $300^{\circ} \mathrm{C}$. Movies were recorded at $125 \mathrm{~Hz}$ with 5 images averaging conducive to minimizing the random noise. So on, the effective frequency was $25 \mathrm{~Hz}$. The whole experimental set up is shown in Fig. 2 and Fig. 3.

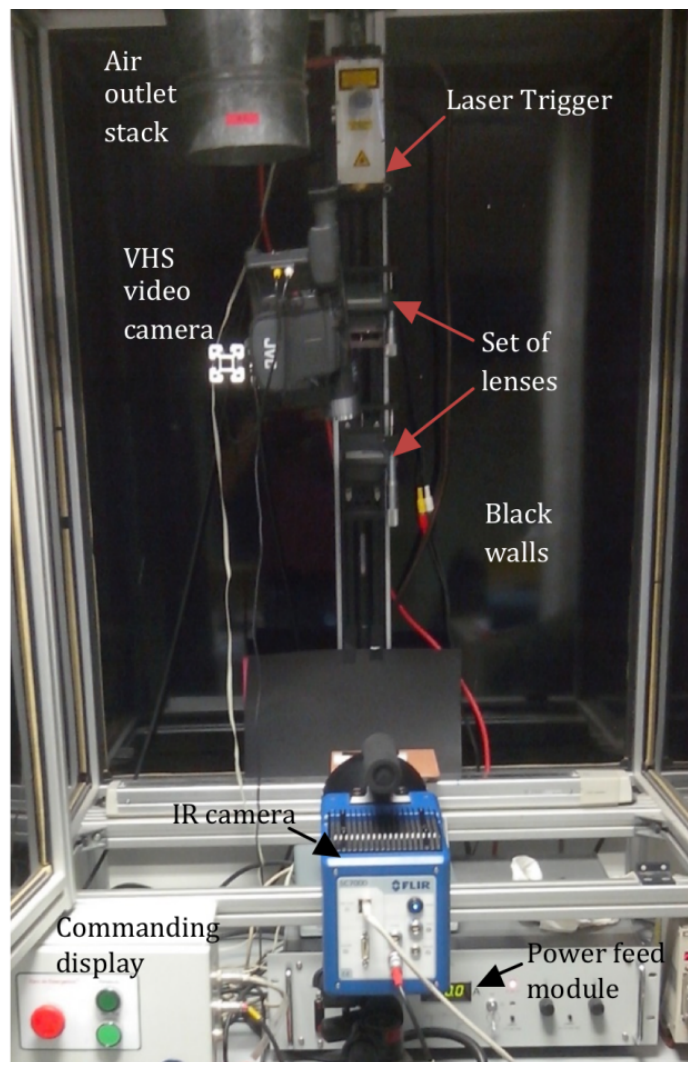

FIGURE 3: Experimental laser device

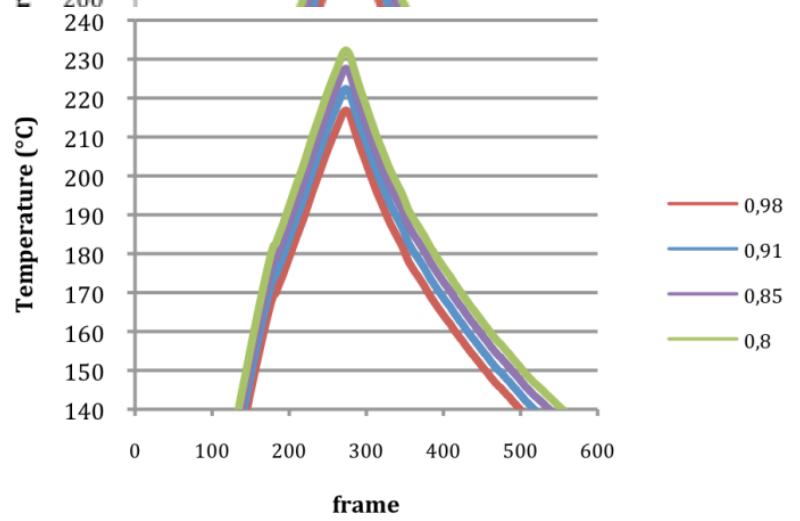

FIGURE 4: Impact of the emissivity variability on maximum temperature 
Infrared measurements are sensitive to the emissivity of the samples. The emissivity was set up as 0.91 for the semi-transparent material and 0.96 for the opaque material; these values are in agreement with previous studies [12]. To estimate the impact of temperature variation due to the emissivity, a variability test was undertaken upon the samples. The emissivity factor varies from 0.8 and 0.98 for a sample irradiated at an energy density level of 22 $\mathrm{J} . \mathrm{mm}^{-2}$. The variation of the maximum temperature is plotted in Fig. 4.

The results are reported in Table 1 , where $\varepsilon=0.91$ is chosen as a reference: Changes in the maximum temperature are within a range of $-5.5^{\circ} \mathrm{C}$ to $9.9^{\circ} \mathrm{C}$. Standard deviation $\sigma$ and average $\mu$ of this data set are $6.44{ }^{\circ} \mathrm{C}$ and $223.46{ }^{\circ} \mathrm{C}$. With these data, the coefficient of variation $\mathrm{Cv}$, calculated with $\mathrm{Cv}=(1+1 /(4 \mathrm{n})) . \sigma / \mu$ is 0.03 , where $\mathrm{n}$ is the population, $\sigma$ is the standard deviation and $\mu$ is the average. Due to the very low value of $\mathrm{Cv}$, the following emissivity coefficients are finally used: 0.91 for transparent material and 0.96 for opaque one.

TABLE 1: Impact of the emissivity variability on temperature measurements

\begin{tabular}{lccccc}
\hline Emissivity $\boldsymbol{\varepsilon}$ & 0.80 & 0.85 & 0.91 & 0.96 & 0.98 \\
\hline Max temperature $\left({ }^{\circ} \mathbf{C}\right)$ & 232.25 & 227.55 & 222.35 & 218.34 & 216.81 \\
\hline$\Delta \mathbf{T}\left({ }^{\circ} \mathbf{C}\right)$ & +9.9 & +5.2 & $/$ & -4.01 & -5.54 \\
\hline
\end{tabular}

\section{RESULTS AND DISCUSSION}

The thermogram of PAEK in Fig. 5 displays a glass transition at $160^{\circ} \mathrm{C}$, followed by a low intensity peak due to water release just after the glass transition, when the macromolecular chains have gained enough mobility to move slightly. Then, a cold crystallization is observed with a maximum at $250^{\circ} \mathrm{C}$ followed by a melting temperature centered at $305^{\circ} \mathrm{C}$. The enthalpy of crystallization $\left(\Delta \mathrm{H}_{\mathrm{c}}=14.9 \mathrm{~J}_{\mathrm{g}}{ }^{-1}\right)$ is lower than the melting enthalpy, $\left(\Delta \mathrm{H}_{\mathrm{m}}=33,7\right.$

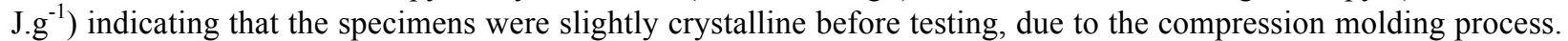
The crystalline rate is usually given by: $\Delta \mathrm{H}_{\mathrm{m}} / \Delta \mathrm{H}_{100 \%}$, but it cannot be calculated since the $\Delta \mathrm{H}_{100 \%}$ is not known due to the novelty of this grade.

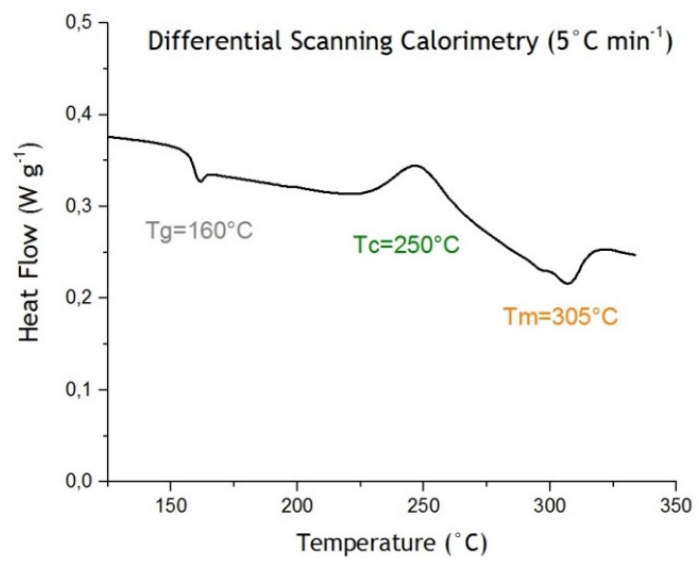

FIGURE 5: DSC thermogram of PAEK for the quasi-amorphous molded specimen

In order to setup all the IR measurements, experiments were firstly performed on a single opaque sample. A power laser measured in Watt is set during a given time (s) upon the spotted area $\left(\mathrm{mm}^{2}\right)$ producing an energy density radiation in $\mathrm{J}_{\mathrm{mm}} \mathrm{m}^{-2}$. Energy density was $22 \mathrm{~J} . \mathrm{mm}^{-2}$ for laser beam/specimen interaction tests. An IR movie was recorded using the parameters defined in the previous section. All the images were processed seeking to match the measured area to the specimen size.

After laser beam/specimen interaction, IR measurements were performed during welding of a couple of $2 \mathrm{~mm}$ thick specimens. A semi-transparent PAEK sample was used as top part, while opaque PAEK was used as bottom one. The energy density was previously determined and fixed at $28 \mathrm{~J}_{\mathrm{mm}} \mathrm{m}^{-2}$ for this test. 
The maximum temperature evolution of the assembly was recorded during welding, with the camera sensor perpendicular to the laser beam direction. The results are plotted in Fig. 6, as well as images at different steps of the process to have a qualitative distribution of the thermal fields. Only temperature above $140^{\circ} \mathrm{C}$ is considered due to the integration time applied. This thermogram confirms that real irradiation time lasts about $15 \mathrm{~s}$. The upper part is semi-transparent to the wavelength: a part of the energy is absorbed by the sample, a part is reflected and the major part of the energy goes through the upper sample to reach the lower part. As the lower part absorbs the laser beam, the energy is stopped at the interface.

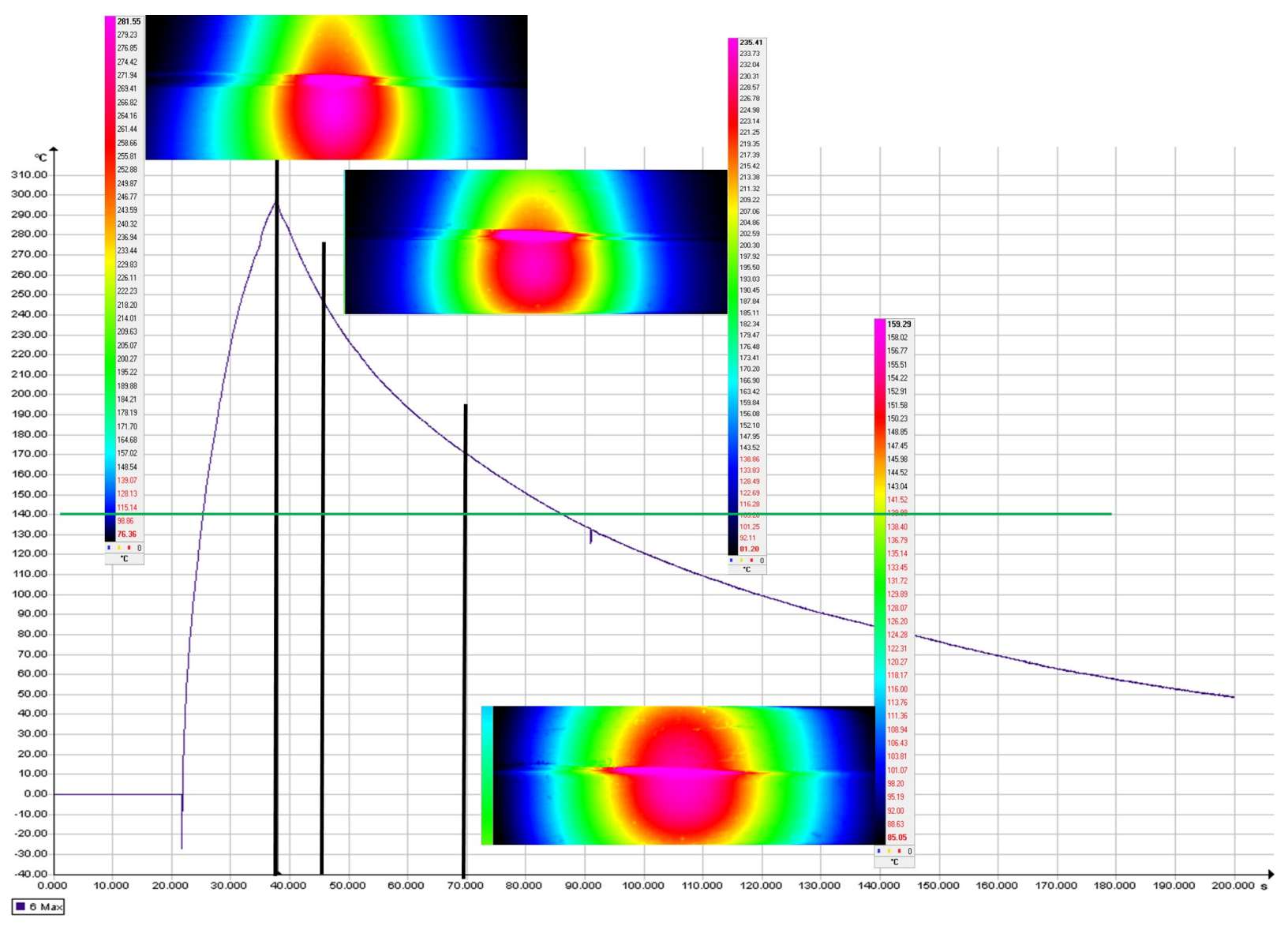

FIGURE 6: IR thermogram for welding test: during and after irradiation of assembly: temperature $\left({ }^{\circ} \mathrm{C}\right)$ versus time (s). The vertical bold black lines indicate the time when the images were recorded.

As a consequence, the temperature at the interface increases up to its maximum at $295+/-7^{\circ} \mathrm{C}$ for an irradiation at $28 \mathrm{~J} \mathrm{~mm}^{-2}$. This temperature is about $130^{\circ} \mathrm{C}$ higher than the glass transition, and reaches the melting temperature. The latter begins at about $275^{\circ} \mathrm{C}$, as seen in Fig. 5. While the upper sample is irradiated, the heat-affected zone is located in the lower sample. As seen in the first image of the temperature field distribution, on the left, the HAZ in the lower part is large and symmetrical. When the irradiation is stopped, the lower part is slightly affected, while the temperature field decreseases in the upper part. Upon cooling, the HAZ in the upper part tremendously stretches out. In all, the temperature at the interface of both samples was higher than the glass transition during $50 \mathrm{~s}$ and higher than the melting temperature during $5 \mathrm{~s}$ for an irradiation of $15 \mathrm{~s}$.

The photograph of the resulting assembly is presented in Fig. 7. The dimensions of the HAZ measured from these pictures are in total agreement with the dimensions obtained by IR thermography. 


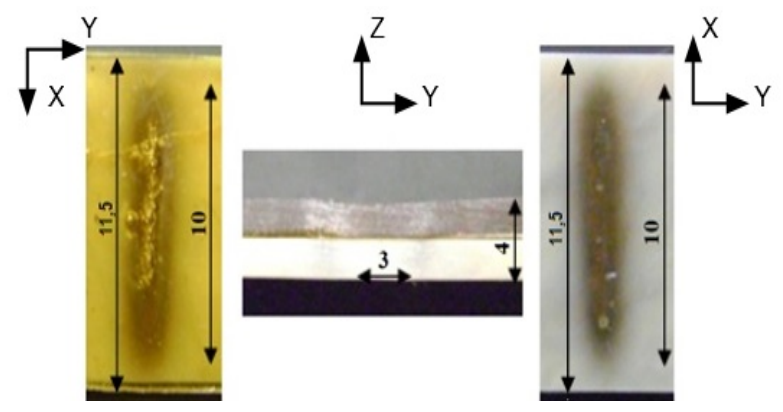

FIGURE 7: Photography of the welded samples: On the left: top view of the assembly, on the middle: side view, on the right: bottom view of the assembly - Dimensions given in $\mathrm{mm}$

\section{CONCLUSION}

The temperature distribution during welding can be measured by infrared thermography with the camera sensor perpendicular to the welded interface. For this study, a high performance thermoplastic of the PAEK family was chosen, its glass transition was measured at $160^{\circ} \mathrm{C}$ and melting range between $275^{\circ} \mathrm{C}$ and $320^{\circ} \mathrm{C}$. The material was processed by compression molding to get semi-crystalline and quasi-amorphous samples.

For the welding test, an assembly made of a quasi-amorphous sample as the upper part and semi-crystalline sample as the lower part was used. The maximum temperature reached at the interface is about $295^{\circ} \mathrm{C}$ when the upper specimen is irradiated for $15 \mathrm{~s}$ with an energy of $28 \mathrm{~J}_{\mathrm{mm}} \mathrm{m}^{-2}$. The temperature inside the sample stays above $\mathrm{Tg}$ during $50 \mathrm{~s}$ and above the melting temperature during $5 \mathrm{~s}$. This time is obviously enough to allow macromolecules to interdiffuse and create entanglements between them. Indeed, the obtained assembly looks strong, although no mechanical test has been performed until now.

This work is a step towards making the transmission laser welding process more reliable. With the chosen energy beam and irradiation time, the maximum temperature inside the sample is kept far from the degradation of PAEK. Moreover, the temperature at the interface reaches the melting temperature thus assuring enough mobility for polymeric chains to get adhesion at the interface. Finally, the location and size of the heat-affected zone (HAZ) has been determined. In future works, the crystalline rate changes and thus, modification of properties in the HAZ will be studied.

\section{ACKNOWLEDGMENTS}

The authors are grateful to the French Ministry of Higher Education and Research for financial support through the funding. of $\mathrm{PhD}$ thesis.

\section{REFERENCES}

1. $\quad$ R. Klein, Laser Welding of Plastics, Wiley VCH, (2012)

2. J.Tarrieu, Ph.D. thesis, Institut National Polytechnique de Toulouse, (2012) (in French)

3. P. G. de Gennes, Comptes Rendus de l'Académie des Sciences, Paris, B291, (1980) (in French)

4. F.Lamethe, Ph.D. thesis, Université Paris VI, (2006) (in French)

5. M. Ilie, J-C. Kneip, S. Matteï, A. Nichici, C. Roze and T. Girasole, Inf. Phys. \& Tech. 51, (2007)

6. M. Speka, S. Matteï, M. Pilloz and M. Ilie, NDT\&E International 41 (2008)

7. G. Gouesbet and G. Gréhan, Atomic Sprays 10 (2000)

8. A.Chateau Akué Asséko, B.Cosson, F.Schmidt, Y.Le Maoult, E.Lafranche, Inf. Phys. \& Tech. 72 (2015)

9. A.Chateau Akué Asséko, B.Cosson, F.Schmidt, Y. Le Maoult, R.Gilblas, E.Lafranche, Inf. Phys. \& Tech. 73 (2015)

10. G. Gouesbet and G. Gréhan, J. of Optics 1 (1999).

11. C.M. Blanca and C. Saloma, Applied Optics, 37 (34) (1998).

12. B. Cosson, F. Schmidt, Y. Le Maoult, M. Bordival. Int J Mater Form 4:1-10 (2011) 